\title{
Effects of application of sucrose and cytokinin to roots on the formation of tuberous roots in sweetpotato (Ipomoea batatas (L.) Lam.)
}

\author{
Toshihiko Eguchi and Satoshi Yoshida \\ Biotron Institute, Kyushu University, Fukuoka 812-8581, Japan \\ Corresponding author: T. Eguchi, E-mail: egut@agr.kyushu-u.ac.jp
}

Received on August 16, 2007; Accepted on February 18, 2008

\begin{abstract}
Effects of sucrose and cytokinin (trans-zeatin riboside [t-ZR]) on the formation of tuberous root of sweetpotato (Ipomoea batatas (L.) Lam.) were studied by directly applying these substances to young roots. In the roots that originally contained approximately $9 \mu \mathrm{g}$ of endogenous sucrose per milligram of fresh weight (FW), further increase in the sucrose level did not necessarily result in tuberous root formation, whereas t-ZR application invariably induced tuberous root formation. However, t-ZR did not induce morphogenesis when the sucrose level in the root was lowered to approximately $2 \mu \mathrm{g} \mathrm{mg}^{-1}$ of FW. Our results imply that high levels of endogenous sucrose are necessary for tuberous root formation and that in the presence of high sucrose concentrations, cytokinins can trigger tuberous root formation.
\end{abstract}

Keywords: cytokinin, sucrose, sweetpotato (Ipomoea batatas (L.) Lam.), tuberous root formation

\section{Introduction}

The tuberous roots of sweetpotato (Ipomoea batatas (L.) Lam.) act as storage organs, which accumulate starch in large quantities. A tuberous root is a portion of root that is thickened locally and is not differentiated into a storage organ as observed in potato (Solanum tuberosum L.) tubers. Anatomical changes during tuberous root formation have already been reported (McCormick 1916, Ogura 1945a,b, Togari 1950, Kokubu 1973, Wilson and Lowe 1973, Lowe and Wilson 1974). This morphogenesis results from active production of storage tissue within the root stele through the proliferation of parenchyma cells. The production of parenchyma cells occurs after development of the primary cambium ring and formation of secondary meristem inside the cambium ring. Therefore, we considered that induction of parenchyma cell proliferation is the first step in the early stages of tuberous root formation.

The exogenous conditions necessary for tuberous root formation have been determined based on previous reports; these include well-aerated conditions in the root zone (Kasugai 1935, Noguchi and Sugawara 1940, Eguchi and Yoshida 2004, Eguchi and Yoshida 2007), dark conditions in the root zone (Kumano and Fujise 1965), and appropriate soil temperature (Eguchi et al. 1994). With regard to the endogenous conditions, a cytokinin named trans-zeatin riboside (t-ZR) (Matsuo et al. 1983, Suye et al. 1983, Sugiyama and Hashizume 1989, Nakatani and Komeichi 1991, Nakatani et al. 2002) and sucrose (Saitou et al. 1997) have been suggested to play important roles in the induction of tuberous root formation. Although cytokinin and/or sucrose levels in roots are considered to regulate the induction of tuberous root formation, the effects of these substances on root thickening of sweetpotatoes are not clarified. It is because the effects of the substances on thickening have been studied by applying them to leaves or a cut-section of stem (Spence and Humphries 1972, McDavid and Alamu 1980, Kadowaki et al. 2000, Kadowaki et al. 2001a, Kadowaki et al. 2001b). In such methods, effects of these substances on root morphogenesis could inseparably include indirect effects through leaf and stem responses to the applied substances. Currently, no information exists regarding the direct effects of local application of sucrose and cytokinins to the roots on the induction of tuberous root formation. One of the reasons for this is the lack of a cultivation technique appropriate to examination the tuberous root formation process. A number of adventitious roots grow from the nodes of sweetpotato plants, and some of these roots develop into tuberous

Eguchi T and Yoshida S 2008 Effects of Application of Sucrose and Cytokinin to Roots on the Formation of Tuberous Roots in Sweetpotato (Ipomoea batatas (L.) Lam.). Plant Root 2: 7-13. doi:10.3117/plantroot.2.7

Copyrights 2008, Plant Root (JSRR), www.plantroot.org 
roots. It is impossible to previously identify the roots which will develop into tuberous roots because root thickening depends on the root zone environment (Togari 1950). We, however, developed a cultivation technique to specify roots that will lead to tuberous root formation (Eguchi and Yoshida 2004). In this study, we verified direct effects of sucrose and cytokinin on tuberous root formation using the cultivation technique.

\section{Materials and Methods}

Growth measurement experiments were carried out using a growth chamber from December 2003 to September 2004, sucrose concentration in the roots was measured from March 2006 to October 2007.

\section{Plant Materials}

The sweetpotato cultivar "Narutokintoki" was used in this study. Cut-stems approximately $25 \mathrm{~cm}$ in length were obtained from plants grown in phytotron at $25 \pm 1{ }^{\circ} \mathrm{C}$ and $70 \pm 5 \%$ relative humidity (RH). Three unfolding leaves (third, forth, and fifth leaves from the apex) were maintained in the cuttings by excising all other leaves. All terminal and axillary buds were excised. Basal parts (approximately $15 \mathrm{~cm}$ in length) of stem cuttings were inserted in wet vermiculite and were grown in phytotron for 10 days. Thereafter, the rooted cuttings were carefully dug out, and used for all experiments.

\section{Growth Conditions}

As shown in Fig. 1, a single nodal root approximately $25 \mathrm{~cm}$ in length on the upper node and several roots on the basal node were maintained in the rooted cutting, which was obtained at 10 days after plantation of cut-stems, by excising all other roots on each node. Plants were transplanted in the hydroponic box for reliable induction of tuberous root formation settled in a growth chamber (Eguchi and Yoshida, 2004); in the box, an aerial space was provided for root swelling above the nutrient solution. The basal half of a single nodal root on the upper node was exposed to air, and the roots on the basal node were completely immersed in the nutrient solution. Plants were grown at $28 \pm 0.5^{\circ} \mathrm{C}$ of air and solution temperature, $70 \pm 3 \% \mathrm{RH}$, $250 \mu \mathrm{mol} \mathrm{m} \mathrm{m}^{-2} \mathrm{~s}^{-1}$ photosynthetic photon flux density (PPFD), and $12 \mathrm{~h}$ photoperiod (0700-1900).

\section{Sucrose and $t-Z R$ Treatments}

The procedure used for the application of sucrose and t-ZR to the roots is shown in Fig. 1. Five days after transplantation (DAT), an approximately 8 -cm-long

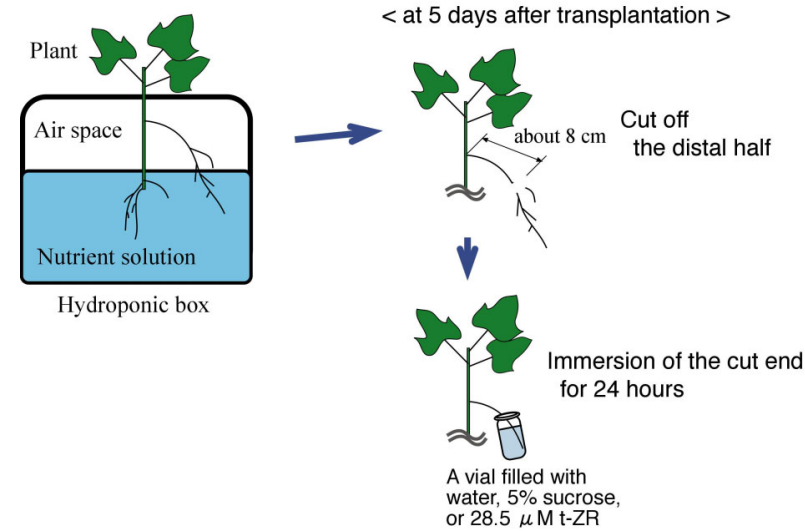

Fig. 1. The procedure of the direct application of water, sucrose, or trans-zeatin riboside (t-ZR) to a sweetpotato root.

root exposed to air (proximal portion) was maintained on the upper node by excising the distal portion with a razor blade. Lateral roots emerging from the nodal root were also excised. The cut end of the nodal root was immediately immersed in $2 \mathrm{~mL}$ of $5 \%$ sucrose solution, $10 \mathrm{ppm}(28.5 \mu \mathrm{M}) \mathrm{t}-\mathrm{ZR}$ solution, or distilled water filled in a glass vial for $24 \mathrm{~h}$. The treatment was conducted under the dark condition to prevent transpiration, which induces excess movement of these solutions to other organs. Six plants were used for each treatment. In order to examine the effect of $t-Z R$ on the roots with lower levels of endogenous sucrose, the plants were grown in dark for 3 days prior to the t-ZR application at 5 DAT. After the t-ZR application, they were grown under the same condition as in the other experiments. Further, a 5\% sucrose solution was applied to a portion of the dark-treated plants for $24 \mathrm{~h}$ just before t-ZR application. Six plants were used for each treatment.

\section{Measurements of Root Growth and Sucrose Content}

Expansive growth of roots treated with sucrose, t-ZR, or water was evaluated by measuring the maximum root diameter. The diameter was measured every 5 days during the 25-day cultivation period. Root sucrose content was measured at transplantation $(0$ DAT), 5 DAT, and at the end of sucrose application, water application, or dark treatment. Six sampled roots were lyophilized immediately. The freeze-dried root (approximately $7 \mathrm{mg}$ ) was ground in a mixer mill (MM200; Retsch, Haan) and was then mixed with 1 $\mathrm{mL}$ deionized water in the mixer mill. The mixture was centrifuged at $10,000 \mathrm{rpm}$ for $10 \mathrm{~min}$, and the supernatant was collected. The residue was mixed with $1 \mathrm{~mL}$ deionized water to re-extract the water-soluble substances and then centrifuged under the same conditions. The re-extraction procedure was 
repeated 3 times, and the extracts were combined and filled up to $5 \mathrm{~mL}$. The extract was filtered through a membrane filter (DISMIIC-25AS; ADVANTEC MFS Inc., Tokyo), and the sucrose in the filtrate was analyzed using a high-performance liquid chromatography (HPLC) system (LC-10AD; SHIMADZU Corp., Kyoto). The filtrate was injected into the HPLC system, and the column was maintained at $40^{\circ} \mathrm{C}$ and was eluted with water at a flow rate of $0.7 \mathrm{~mL} \mathrm{~min}^{-1}$.

\section{Microscopic Observation}

The anatomical features of the nodal root were observed after a 25-day cultivation period. Hand-cut cross-sections were obtained from the thickest root region. These sections were stained with $0.02 \%$ toluidine blue or $2 \%$ Phloroglucinol-alcohol solution, and observed under optical microscopes (OPTIPHOT and SMZ1000; Nikon, Tokyo). Toluidine blue-stained sections were used to observe the primary cambium and secondary meristematic tissue of the root. The developmental stages of the primary cambium were classified into 3 stages based on the shape: stage A, several primary cambia were dispersedly located; stage $\mathrm{B}$, primary cambia congregated together to form an irregular circle; and stage $\mathrm{C}$, the primary cambium ring was clearly formed. We also observed the secondary meristematic tissue formed within the region encircled by the circular primary cambium ring. The phloroglucinol-stained sections were used for evaluating the degree of lignification within the region encircled by the primary cambium. The degree was calculated as an areal ratio of the red-stained cells to the region encircled by the primary cambium.

\section{Results}

\section{Sucrose Content}

The sucrose contents of the roots at 0 DAT, 5 DAT, and just after water application were $7.8 \mu \mathrm{g} \mathrm{mg}^{-1}, 9.1$ $\mu \mathrm{g} \mathrm{mg}^{-1}$, and $8.0 \mu \mathrm{g} \mathrm{mg}^{-1}$, respectively, and there were no significant differences among these values (Fig. 2). The application of $5 \%$ sucrose solution to the root increased the sucrose content to $13.1 \mu \mathrm{g} \mathrm{mg}^{-1}$ after 24 $\mathrm{h}$, which was $144 \%$ of that before treatment. The 3-day dark treatment prior to the t-ZR application decreased the sucrose content to $2.3 \mu \mathrm{g} \mathrm{mg}^{-1}$, which was only $25 \%$ of that at 5 DAT in the non-dark-treated plants. Sucrose application to the dark-treated plants increased the sucrose content of the roots. However, the content was only $38 \%$ of that in non-dark treated and non-sucrose-applied plants.

\section{Root Growth}

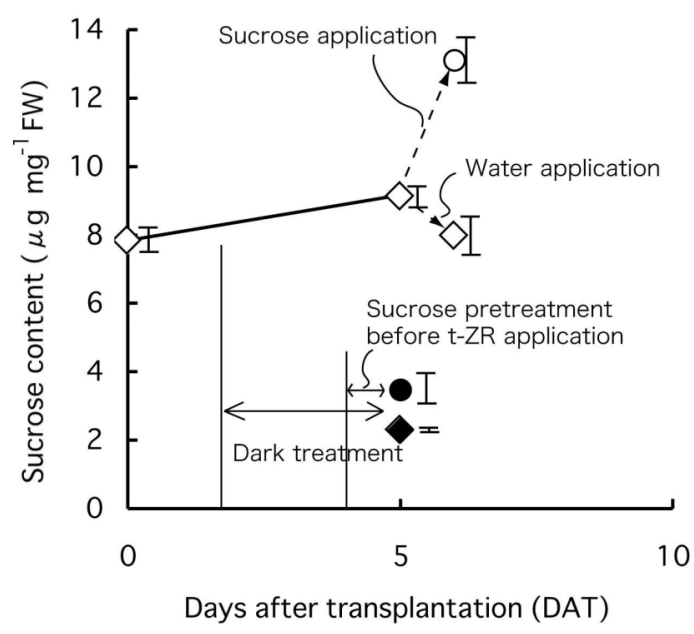

Fig. 2. Changes in sucrose content in the roots after transplantation. Plants were grown under a $12 \mathrm{~h}$ light photoperiod (open symbols), or under continuous darkness for 3 days prior to t-ZR application of a growth regulator (closed symbols). Vertical bars indicate SE.

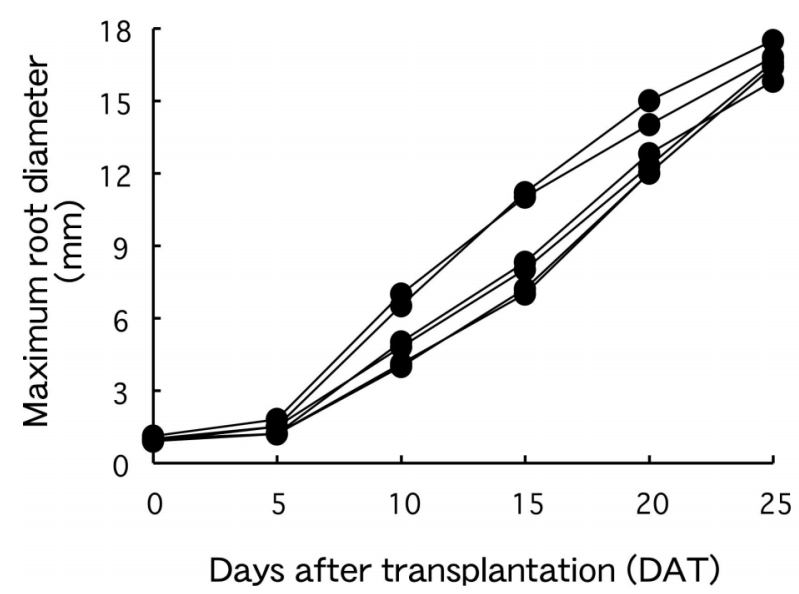

Fig. 3. Changes in the maximum diameter of the "intact" roots on the upper node. The changes were depicted every plant.

When the root on the upper node was maintained "intact" (i.e., no substances were applied to it and the distal half was not cut), the portion exposed to air swelled vigorously and developed into a tuberous root (Fig. 3). In all plants, the root diameter exceeded 15 $\mathrm{mm}$ at the end of the 25-day cultivation. The thickest part of the root displayed anatomical features similar to those of a tuberous root, which were the active primary cambium ring and the secondary meristem formed within the cambium ring. Thus, the cultivation method was considered satisfactory for the exogenous conditions necessary for the induction of tuberous root formation. 
(a)

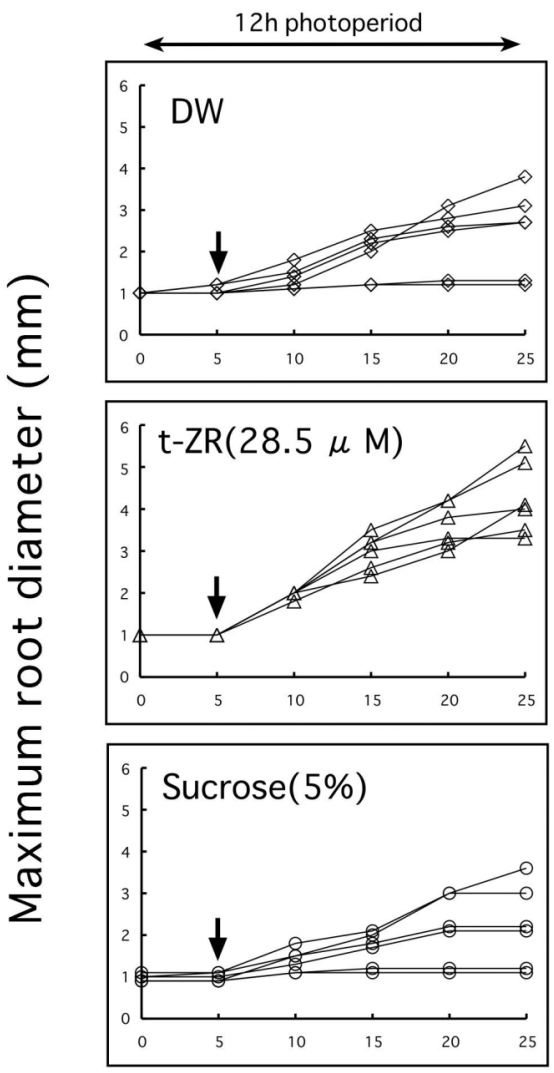

(b)
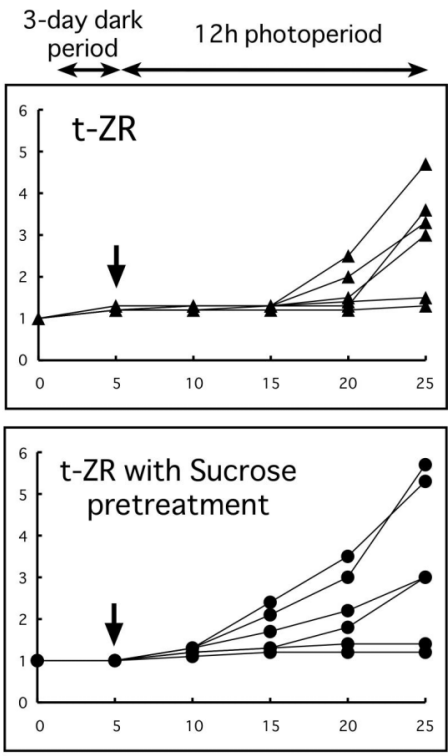

Days after transplantation (DAT)

Fig. 4. Changes in the maximum diameter of the roots applied with distilled water (DW), trans-zeatin riboside (t-ZR), or sucrose. Plants were grown under a $12 \mathrm{~h}$ light photoperiod (a) and under continuous darkness for 3 days prior to t-ZR applications (b). Arrows show the time when DW, t-ZR, and sucrose were applied.

Figure $4 \mathrm{a}$ shows the time-course changes in root diameter when water, sucrose, and t-ZR were applied. During application, these solutions in the vials were decreased by more than $0.15 \mathrm{~mL}$ for all roots. This decrement was considered to be almost equal to the amount of solution taken up by the plant, because water loss by evaporation was not observed in the control vials in which the root end was not inserted. The diameters at 25 DAT did not correlate with the amount of solution taken up by the roots in all the treatments (data not shown). Only the t-ZR treatment produced an obvious response to the application, and the maximum diameter of the roots exceeded $3 \mathrm{~mm}$ at 25 DAT in all plants. Results of sucrose application were similar to those of water application: at 25 DAT, the diameter of one-third of the roots scarcely increased, and another one-third of the roots reached 3 $\mathrm{mm}$ in diameter. Figure $4 \mathrm{~b}$ shows the time-course changes in the diameter of the t-ZR-treated roots in the dark-treated plants for which the endogenous sucrose level was low (Fig. 2). The diameter of the non- sucrose-pretreated roots scarcely increased over 10 days after the t-ZR treatment in all plants; thereafter, one-third of the roots remained thin, and the diameter of the others increased up to $3 \mathrm{~mm}$. The sucrose-pretreated roots showed little swelling after more than 5 days of the t-ZR treatment, and the subsequent changes in root diameter were similar to the results of the water and sucrose application to non-dark-treated plants (Fig. 4a), where the diameter of one-third of the roots scarcely increased and that of another one-third of the roots exceeded $3 \mathrm{~mm}$ at 25 DAT. In non-dark-treated plants, all t-ZR-treated roots attained stage $\mathrm{C}$; however, no plants treated with sucrose had roots that reached stage $C$, and only 2 plants treated with water had roots that reached stage C (Fig. 5). Formation of the secondary meristem within the root stele was observed in roots that swelled to a diameter greater than $2.7 \mathrm{~mm}$. The percentage of the roots that formed the secondary meristem was $67 \%, 33 \%$, and $100 \%$ in the roots treated with water, sucrose, and t-ZR, respectively. One-third of the roots 
treated with water or sucrose continued to form the secondary meristem at stage B. Figure 6 shows the relationship between the root diameter and the degree of the lignified region within the root stele of non-dark-treated plants. The degree of lignification decreased with an increase in the root diameter and decreased below $10 \%$ in roots with diameters more than $3 \mathrm{~mm}$. Raise of sucrose level resulted in heavy lignification of thin roots that scarcely swelled over the 25-day cultivation. There was no correlation between the amount of sucrose solution uptake and the degree of lignification (data not shown).

\section{Discussion}

Sucrose and cytokinin (t-ZR) were locally applied to the portion of young root exposed to air. In roots with a sucrose level of approximately $9 \mu \mathrm{g} \mathrm{mg}{ }^{-1}$, further increase in the sucrose level did not necessarily result in tuberous root formation, while t-ZR application invariably induced tuberous root formation. The application of t-ZR, however, did not affect root swelling when the level of sucrose in the root was

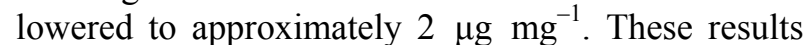
imply that a high level of endogenous sucrose is necessary for tuberous root formation, and in this condition, exogenously applied cytokinins can trigger tuberous root formation. In the formation process, stage $\mathrm{C}$ is considered to occur in the early stage when the secondary meristem forms in the region encircled by the primary cambium ring (Eguchi and Yoshida, 2004). Secondary meristem formation, however, continued in the roots in stage B in the case of sucrose and water application treatments. Exogenous cytokinins may affect the development of the primary cambium, and the formation of the secondary meristem may be regulated through other processes.

In cross-sections of roots developing to tuberous root, parenchyma within the stele increases the area with root swelling (Eguchi et al. 2004), and ratio of lignified region within the primary cambium ring decreases during the early stages of tuberous root formation (Eguchi and Yoshida 2007). Induction of tuberous root morphogenesis and the subsequent expansion are both inhibited due to $\mathrm{O}_{2}$ deprivation, and the stress promotes lignification within the root stele (Eguchi and Yoshida 2007). In this study, high sucrose level also promoted lignification of roots that remained thin over the 25-day cultivation. We found no correlation between the amount of sucrose uptake and the degree of lignification; however, these roots appeared to be damaged due to high sucrose concentration.

Endogenous conditions necessary for the induction of tuberous root formation resemble those required for the activation of CycD3, a D-type cyclin. CycD3 may

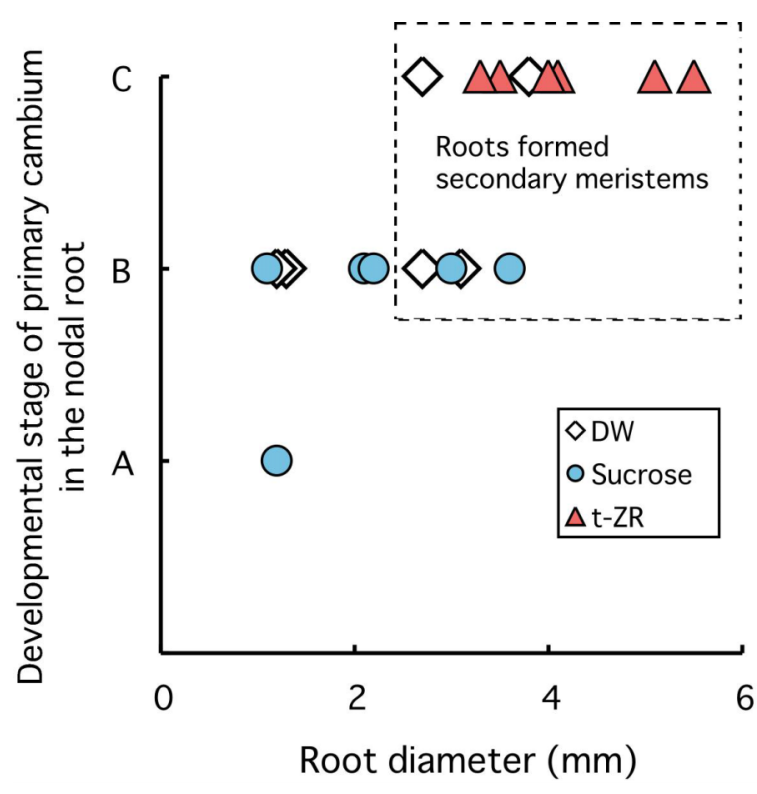

Fig. 5. Developmental stage and root diameter at 25 days after transplantation (DAT). Roots were treated with distilled water (DW), trans-zeatin riboside (t-ZR), or sucrose. Development of the root primary cambium was classified into 3 stages: stage A, several primary cambia were dispersedly located; stage B, the primary cambia congregated together to form an irregular circle; and stage $\mathrm{C}$, a primary cambium ring developed to form a circular shape. Roots forming secondary meristems are enclosed within a dashed rectangular box.

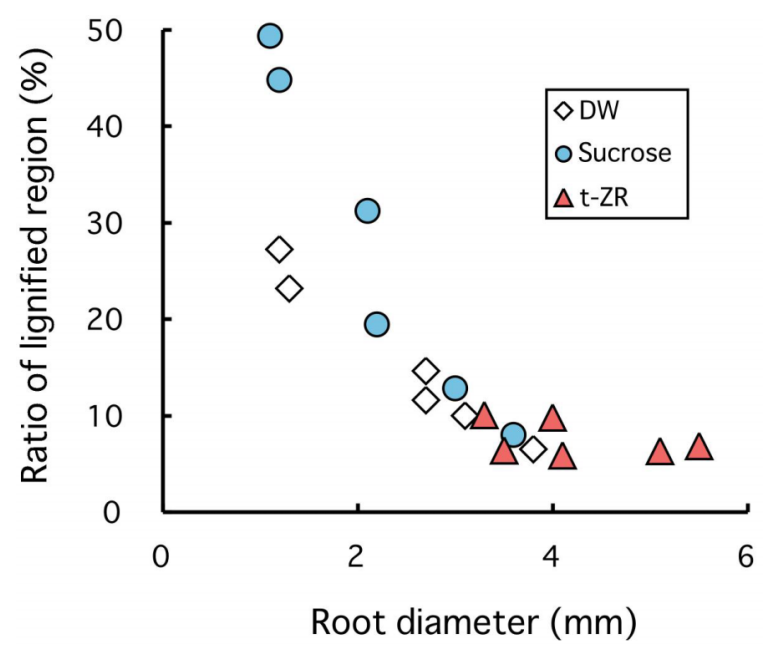

Fig. 6. Ratio of the lignified region within the root to the root diameter at 25 days after transplantation (DAT). Roots were treated with distilled water (DW), trans-zeatin riboside (t-ZR), or sucrose.

dominantly drive the G1/S transition in the cell division cycle (Menges et al. 2006). In Arabidopsis thaliana, cytokinins induce cell division through the activation of CycD3 (Riou-Khamlichi et al. 1999), but $\mathrm{CycD} 3$ is not present under sucrose starvation conditions (Sandra Healy et al. 2001). Indeed, the transcript 
level of CycD3 in sweetpotato root increased at an early stage of tuberous root formation (Nagata et al. 2004). Furthermore, sucrose application to a sweetpotato petiole, not to a root, increased the $\mathrm{CycD} 3$ level in the petiole, where the simultaneous application of sucrose and cytokinins further increased the CycD3 level (Nagata et al. 2005). Sucrose level in young roots also positively regulates the sucrose synthase activity, which is closely related to sugar import, starch synthesis, cell wall synthesis, and sink activity (Saitou et al. 1997). Endogenous sucrose level may relate not only proliferation of parenchyma cells but also differentiation of these cells to storage cells throughout tuberous root development. Both sucrose and cytokinins may play key roles in the induction of tuberous root formation in sweetpotato through the proliferation of parenchyma cells; therefore, future expression analyses of the genes related to tuberous root formation should preferably be carried out using a cultivation method that allows for control of tuberous root formation, such as the method used in this study.

Although our results imply that cytokinins induce tuberous root formation, no information exists regarding the changes in the level of endogenous cytokinins in the portion of roots in the initial stages of tuberous root development. This is because previous studies (Matsuo et al. 1983, Nakatani et al. 1991) were carried out using roots with uncertain fates or roots that were already in the early stages of tuberous root formation. Thus, the time-course pattern of cytokinin levels should be reconfirmed in the root portions that have been definitely determined to develop into tuberous roots. Furthermore, simultaneous changes in the levels of cytokinins in the distal portion of the root should also be investigated in order to elucidate the contribution of translocated cytokinins to the induction of tuberous root formation. Root tips are the major sites of cytokinin biosynthesis, and are also the source of the plant hormone that is translocated via the xylem (Letham 1994); however, in this study, root tips were absent in the roots treated with growth substances. Therefore, the cytokinin-treated root was provided with the artificial source of translocated cytokinins. Cytokinins may merely act as paracrine signals with respect to apical dominance and leaf senescence (Faiss et al. 1997), while the hormone of root origin may act as a endocrine signal for nitrogen metabolism in maize leaves (Sakakibara et al. 1998, Takei et al. 2001) or adventitious root formation in cucumber hypocotyls (Kuroha et al. 2002). Our results suggest that tuberous root formation can be induced by the translocated cytokinins; however, extensive research is necessary to elucidate the physiological role of the translocated cytokinins in the morphogenesis.

\section{References}

Eguchi T, Kitano M, Eguchi H 1994 Effect of root temperature on sink strength of tuberous root in sweet potato plants (Ipomoea batatas Lam.). Biotronics 23: 75-80.

Eguchi T, Yoshida S 2004 A cultivation method to ensure tuberous root formation in sweetpotatoes (Ipomoea batatas (L.) Lam.). Environ. Control in Biol. 42: 259-266.

Eguchi T, Yoshida S 2007 Effects of gas exchange inhibition and hypoxia on tuberous root morphogenesis in sweetpotato (Ipomoea batatas (L.) Lam.). Environ. Control in Biol. 45: 103-111.

Faiss M, Zalubilova J, Strnad M, Schmulling T 1997 Conditional transgenic expression of ipt gene indicates a function for cytokinins in paracrine signaling in whole tobacco plants. Plant J. 12: 401-415.

Healy JMS, Menges M, Doonan JH, Murray JAH 2001 The Arabidopsis D-type cyclins $\mathrm{CycD} 2$ and CycD3 both interact in vivo with the PSTAIRE cyclin-dependent kinase Cdc2a but are differentially controlled. J. Biol. Chem. 276: 7041-7047.

Kadowaki M, Kubota F, Saitou K 2000 Effects of exogenous injection of different sugars on leaf photosynthesis, dry matter production and adenosine 5'-diphosphate glucose pyrophosphorylase (AGPase) activity in sweet potato, Ipomoea batatas (Lam.). J. Agronomy and Crop Sci. 186: $37-41$.

Kadowaki M, Kubota F, Saitou K 2001a Effects of exogenous injection of sucrose solution to plant on the carbon distribution to tuberous root and tuberous root production in sweet potato, Ipomoea batatas Lam. Jpn. J. Crop Sci. 70: 575-579. (in Japanese with English abstract)

Kadowaki M, Murayama N, Kubota F 2001b Effect of sucrose solution injection at different growth stages on production and ADP-glucose pyrophosphorylase activity of roots in sweet potato, Ipomoea batatas Lam., cultivars. J. Fac. Agr., Kyushu Univ. 47: 13-20.

Kasugai S 1935 Hydroponics for sweet potatoes. Jpn. J. Crop Sci. 7: 12-20. (in Japanese)

Kokubu T 1973 Thremmatological studies on the relationship between the structure of tuberous root and its starch accumulating function in sweet potato varieties. Bull. Fac. Agr., Kagoshima Univ. 22: 1-126. (in Japanese with English abstract)

Kumano S, Fujise K 1965 Influence of environmental conditions of roots on the tuberous root formation of sweet potato. Jpn. J. Crop Sci. 34: 35-39. (in Japanese with English abstract)

Kuroha T, Kato H, Asami T, Yoshida S, Kamada H, Satoh S 2002 A trans-zeatin riboside in root xylem sap negatively regulates adventitious root formation on cucumber hypocotyls. J. Exp. Bot. 53: 2193-2200.

Letham DS 1994 Cytokinins as phytohormones: sites of biosynthesis, translocation, and function of translocated cytokinin. In: Mok DWS, Mok MC, Eds., Cytokinins: chemistry, activity, and function. CRC Press, Boca Raton, pp. $57-80$.

Lowe SB, Wilson LA 1974 Comparative analysis of tuber development in six sweet potato (Ipomoea batatas (L.) Lam.) cultivars. 1. Tuber initiation, tuber growth and partition of assimilate. Ann. Bot. 38: 307-317.

Matsuo T, Yoneda T, Itoo S 1983 Identification of free cytokinins and the changes in endogenous levels during tuber development of sweet potato (Ipomoea batatas Lam.). Plant Cell Physiol. 24: 1305-1312. 
McCormick FA 1916 Notes on the anatomy of the young tuber of Ipomoea batatas Lam. Bot. Gaz. 61: 388-398.

McDavid CR, Alamu S 1980 The effect of growth regulators on tuber initiation and growth in rooted leaves of two sweet potato cultivars. Ann. Bot. 45: 363-364.

Menges M, Samland AK, Planchais S, Murray JAH 2006 The D-type cyclin CYCD3;1 is limiting for the G1-to-S-phase transition in arabidopsis. Plant Cell 18: 893-906.

Nagata T, Saitou K, Fukuda K 2004 Molecular cloning and expression analysis of a gene for a cyclin D3 in sweet potato (Ipomoea batatas Lam.). Jpn. J. Crop Sci. 73 (Extra issue 2): 368-369. (in Japanese)

Nagata T, Saitou K, Toriyama H, Yamada M, Kubota K 2005 Expression of two distinct cycline D3 genes in sweet potato (Ipomoea batatas Lam.). Jpn. J. Crop Sci. 74 (Extra issue 2): 112-113. (in Japanese)

Nakatani M, Komeichi M 1991 Changes in endogenous level of zeatin riboside, abscisic acid and indole acetic acid during formation and thickening of tuberous root in sweet potato. Jpn. J. Crop Sci. 60: 91-100. (in Japanese with English abstract)

Nakatani M, Tanaka M, Yoshinaga M 2002 Physiological and anatomical characterization of a late-storage root-forming mutant of sweetpotato. J. Am. Soc. Hortic. Sci. 127: 178-183.

Noguchi Y, Sugawara T 1940 A study on mechanism of tuberous root formation in sweet potatoes. (in Japanese; title translated into English by present authors) Agric. Hortic. 15: 1-8.

Ogura K 1945a Anatomical studies on tuberous root formation in sweet potatoes [1]. Agric. Hortic. 20: 331-334. (in Japanese)

Ogura K 1945b Anatomical studies on tuberous root formation in sweet potatoes [2]. Agric. Hortic. 20: 381-383. (in Japanese)

Riou-Khamlichi C, Huntly R, Jacqmard A, Murray JAH 1999 Cytokinin activation of Arabidopsis cell division through a D-type cyclin. Science 283: 1541-1544.

Saitou K, Araki T, Agata W, Kubota F, Nakayama K 1997 Expression of sucrose synthase in sweet potato. Jpn. J. Crop Sci. 66: 624-631. (in Japanese with English abstract)
Sakakibara H, Suzuki M, Takei K, Deji A, Taniguchi M, Sugiyama T 1998 A response-regulator homologue possibly involved in nitrogen signal transduction mediated by cytokinin in maize. Plant J. 14: 337-344.

Spence JA, Humphries EC 1972 Effect of moisture supply, root temperature, and growth regulators on photosynthesis of isolated rooted leaves of sweet potato (Ipomoea batatas). Ann. Bot. 36: 115-121.

Sugiyama T, Hashizume T 1989 Cytokinins in developing tuberous roots of sweet potato. Agric. Biol. Chem. 53: $49-52$.

Suye S, Sugiyama T, Hashizume T 1983 Mass spectrometric determination of ribosyl trans-zeatin from sweet potato tubers (Ipomoea batatas L. cv. Kohkei No. 14). Agric. Biol. Chem. 47: 1665-1666.

Takei K, Sakakibara H, Taniguchi M, Sugiyama T 2001 Nitrogen-dependent accumulation of cytokinins in root and the translocation to leaf: implication of cytokinin species that induces gene expression of maize response regulator. Plant Cell Physiol. 42: 85-93.

Togari Y 1950 A study of tuberous root formation in sweet potato. Bull. Nat. Agr. Exp. Station 68: 1-96. (in Japanese with English abstract)

Wilson LA, Lowe SB 1973 The anatomy of the root system in West Indian sweet potato (Ipomoea batatas (L.) Lam.) cultivars. Ann. Bot. 37: 633-643.

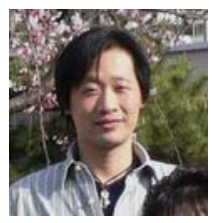

Dr. Toshihiko Eguchi's research interests are regulatory processes of storage organ morphogenesis in root and tuber crops as affected by environments.

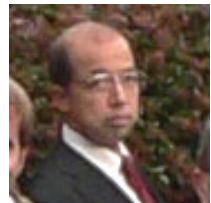

Dr. Satoshi Yoshida's research interests concern plant environment control both for horticultural crop production in a greenhouse and for experimental cultivation in a laboratory. 